\title{
ИНТЕНСИВНАЯ ТЕРАПИЯ: КРАТКАЯ ИСТОРИЯ СТАНОВЛЕНИЯ, СОВРЕМЕННОЕ ЗНАЧЕНИЕ, ПРОБЛЕМЫ И ПЕРСПЕКТИВЫ В ОБОЗРИМОМ БУДУЩЕМ
}

\author{
В. А. РУДНОВ \\ 'ФГБОУ ВО «Уральский государственный медицинский университет» МЗ РФ, г. Екатеринбург, РФ \\ ²МАЗ «Городская клиническая больница № 40», г. Екатеринбург, РФ
}

Показана эволюция становления реанимации и интенсивной терапии в мире и в России и роль отдельных специалистов в ее формировании и развитии. Продемонстрировано значение специальности и службы в современной клинической медицине. Отмечено, что роль медицины критических состояний с течением времени возрастает. Будет наблюдаться рост числа коек интенсивного этапа оказания помощи в стационарах, возникнет необходимость в структурировании отделений интенсивной терапии и реанимации в зависимости от тяжести состояния пациентов и объема оказания помощи. Следует констатировать более рациональное отношение врачей к использованию многих лечебно-диагностических методов и лекарственных средств. Среди обозримых перспектив развития специальности необходимо выделить определение ее финансирования в соответствии с затратами, создание в лечебно-профилактических учреждениях группы ранней мобилизации и реабилитации, улучшение профилактики инфекционных осложнений.

Ключевые слова: история, интенсивная терапия, проблемы, перспективы

Для цитирования: Руднов В. А. Интенсивная терапия: краткая история становления, современное значение, проблемы и перспективы в обозримом будущем // Вестник анестезиологии и реаниматологии. - 2019. - Т. 16, № 2. - C. 15-24. DOI: 10.21292/2078-5658-2019-16-2-15-24

\section{INTENSIVE CARE: BRIEF HISTORY OF ESTABLISHMENT, CURRENT SIGNIFICANCE, PROBLEMS AND PERSPECTIVES IN THE NEAR FUTURE}

\section{A. RUDNOV 1,2}

1Ural State Medical University, Yekaterinburg, Russia

${ }^{2}$ Municipal Clinical Hospital no. 40, Yekaterinburg, Russia

The article describes the evolution of resuscitation and intensive care establishment in the world and Russia and the role of individual specialists in its formation and development. The importance of this specialty and services in modern clinical medicine is demonstrated. It is noted that the role of critical medicine is increasing over time. There will be an increase in the number of intensive care beds in hospitals, there will be a need to structure the intensive and critical care units, depending on the severity of patients and scope of provided care. It worth mentioning that doctors developed a more rational attitude to the use of many therapeutic and diagnostic tools and medicines. Regarding nearest development prospects of this field of medicine, it is necessary to highlight that it is to be funded based on costs, early mobilization and rehabilitation groups are to be organized in medical units, and prevention of infectious complications is to be improved.

Key words: history, intensive care, problems, perspectives

For citations: Rudnov V.A. Intensive care: brief history of establishment, current significance, problems and perspectives in the near future. Messenger of Anesthesiology and Resuscitation, 2019, Vol. 16, no. 2, P. 15-24. (In Russ.) DOI: 10.21292/2078-5658-2019-16-2-15-24

Интенсивной терапии и реаниматологии минуло уже 65 лет. Понимая роль и значимость ее фундаментальной основы - анестезиологии, существующую общность специальности в нашей стране, мы, тем не менее, посвящаем эту лекцию исключительно разделу интенсивной терапии и реаниматологии, при этом ни в коей мере не стремясь и не предлагая разделение специальностей. Время и конкретная ситуация, которая будет складываться в клинической медицине, покажут ракурс дальнейшего движения. Мы полагаем, что польза от прочтения данной публикации заключается прежде всего в осознании главных направлений развития интенсивной терапии, концентрации на них повседневных моральных и физических затрат персонала и, что также немаловажно, финансовых ресурсов. Наша цель - изложение основных исторических вех специальности, понимание ее настоящего значения в клинической медицине и ближайших перспектив.
Терминология. Сложившаяся на настоящий момент терминология оперирует следующим понятийным аппаратом: медицина критических состояний (Critical care medicine, USA), интенсивная терапия /реанимация/интенсивная помощь - в ряде европейских стран (Intensive care medicine, Intensive care and reanimation, Soins intensive et reanimation). В России в настоящее время официально специальность одна - «анестезиология-реаниматология», хотя в реальной практике, наряду с отделениями анестезиологии-реанимации (ОАР), существуют отделения реанимации и интенсивной терапии (ОРИТ), включая специализированные. Среди них следует упомянуть отделения нейрореанимации, акушерские, неонатальные, инфекционные, заместительной почечной терапии, а также блоки в инфарктных и пульмонологических отделениях. Жизнь распорядилась так, что в лечебных учреждениях в зависимости от существующего потока пациентов происходило формирование того или иного 
типа отделений - поливалентных или специализированных. В самом общем виде под интенсивной терапией понимают комплекс мер, направленных на предупреждение или устранение расстройств жизненно важных функций, возникших в связи с заболеванием, травмой или операцией.

История появления и развития отделений интенсивной терапии. Принято считать, что впервые идею концентрации тяжелораненых в одном помещении для облегчения ухода и контроля ситуации подала английский общественный деятель, медсестра по образованию, Флоренс Найтингел в середине 50-х годов XIX в. в период Крымской войны, когда газеты были полны сообщений о необоснованно высокой смертности среди военнослужащих. В последующие годы идея не получила поддержки, и причиной тому служит политическая нестабильность в Европе и, по-видимому, уровень развития медицины в большинстве стран. Вероятной предтечей современных отделений реанимации следует считать сформулированную и реализованную на практике в 1923 г. американским нейрохирургом W. Dandy концепцию послеоперационного восстановления оперированных пациентов в условиях отдельного подразделения, которым занималась команда специально подготовленных медсестер. Чуть позднее, в 1930 г., в университетской хирургической клинике Тюбингена мы встречаем уже пример, свидетельствующий о целесообразности создания комбинированного послеоперационного отделения для пациентов, подвергнутых оперативному лечению, и отделения интенсивной терапии. Инициатором создания такого подразделения был известный в Германии хирург Мартин Киршнер [44]. Не исключается, что подобная практика в единичном режиме имела место и в других странах, однако история медицины не сохранила письменных доказательств. Мы можем утверждать, что локальные, хотя и позитивные результаты W. Dandy и M. Kirschner указывали на целесообразность создания подобных отделений, однако в реальной жизни не отмечалось расширения внедрения в практику других лечебных учреждений данной организационной технологии. И только спустя 30 лет прогрессивная идея создания ОРИТ получает свое практическое воплощение. Мощным стимулом по ее реализации послужила эпидемия полиомиелита в Дании. Когда в различные больницы датского королевства поступило 316 пациентов, требующих проведения искусственной вентиляции легких (ИВЛ) вследствие паралича дыхательных мышц и/или бульбарного синдрома, которую проводили вручную, сменяя друг друга на протяжении длительного времени (несколько недель) более 1000 студентов медицинских вузов, дантистов, медиков-волонтеров и служителей культа. Эта эпидемия полиомиелита, осложненного острой дыхательной недостаточностью (ОДН), и послужила основанием целесообразности открытия отделений нового типа и создания респираторов-автоматов. И, как ока- залось, она положила начало новой медицинской специальности [3, 16, 30, 46].

Считается, что основателем первого в мире ОРИТ в Копенгагене в декабре 1953 г. был 38-летний датский анестезиолог Bjorn Aage Ibsen. Первым же пациентом этого отделения оказался 43-летний мужчина, поступивший из палаты наблюдения после странгуляционной механической асфиксии с проявлениями энцефалопатии и ОДН. При рентгенологическом исследовании органов грудной клетки в легких определялись двухсторонние билатеральные инфильтраты и признаки отека. Пациенту проводили ИВЛ сначала через маску мешка Амбу. Затем он был интубирован, и вентиляцию легких осуществляли через эндотрахеальную трубку тем же мешком вручную. В качестве газовой смеси использовали комбинацию $\mathrm{O}_{2}$ и $\mathrm{N}_{2} \mathrm{O}$ в соотношении 60/40. Интересно, что уже тогда ему проводили мониторинг сатурации гемоглобина крови с помощью аппарата Millikan Oximeter, измеряли КОС. Конечно, вызывает вопросы проводимая медикаментозная терапия, но она отражает представления того времени о медицине критических состояний. Пациент получал трансфузию 500 мл крови, инфузию 1000 мл изотонического раствора глюкозы, антибиотик ауреомицин по 250 мг $\times 4$ раза в сутки, теофиламин 400 мг/сут и прокаин 100 мг/сут. Авторы не сообщили об исходе у этого пациента, но указывают на то, что он был излишне рано экстубирован и возвращен в терапевтическое отделение. Однако последующее течение его патологического процесса потребовало повторной госпитализации в отделение реанимации, интубации, возобновления ИВЛ и трахеостомии [14].

Основателем медицины критических состояний и реаниматологии в России можно в полной мере считать академика В. А. Неговского, который в 1936 г. организовал экспериментальную лабораторию по оживлению, а на полях Второй мировой войны способствовал появлению противошоковых палаток, где оказывалась помощь тяжелораненым, включая внутриартериальное введение солевых растворов и крови. В дальнейшем школа В. А. Неговского разработала концепцию постреанимационной болезни, методологические основы дефибрилляции сердца [2]. Немалая роль в становлении специальности принадлежит и хирургам. В частности, в 1958 г. по инициативе П. А. Куприянова была открыта первая в стране кафедра анестезиологии, ставшая базой для подготовки высокопрофессиональных специалистов как в армии, так и в гражданском здравоохранении. Кафедра служила плацдармом для преподавания основ и новаций в области анестезиологии и реаниматологии. Благодаря ее появлению мы узнали такие имена, как Б. С. Уваров, Ю. Н. Шанин, А. Л. Костюченко, Ю. С. Полушин, А. И. Левшанков и др. Было бы большой несправедливостью не упомянуть имена тех, кто внес серьезный вклад в развитие различных аспектов интенсивной терапии, работая в Москве и распространяя 
передовые технологии в различных регионах страны: Р. Н. Лебедева, В. А. Гологорский, Г. А. Рябов, В. Л. Кассиль, А. З. Маневич, Б. Р. Гельфанд. Особая роль в популяризации и развитии специальности принадлежит А. П. Зильберу.

Согласно Большой медицинской энциклопедии, в 1959 г. в больнице им. С. П. Боткина было открыто по сути первое в нашей стране самостоятельное реанимационное отделение - клиническая база научно-исследовательской лаборатории общей реаниматологии АМН СССР (акад. В. А. Неговский) при госпитальной хирургической клинике 1-го ММИ и НИИ клинической и экспериментальной хирургии М3 СССР (акад. Б. В. Петровский).

Таким образом, мы можем констатировать, что реаниматология и понимание необходимости создания отделений реанимации в России сформировались почти одновременно с ведущими европейскими странами и США, имея свой приоритет в области экспериментальных исследований. Между тем будет уместно вновь вернуться в 50-е годы, отметив еще ряд важных вех. В одинаковой степени это касается Макса Вейля и Питера Сафара. Если первый является основателем медицинского центра критических состояний в Южной Калифорнии (г. Лос-Анжелес), разработавшим основы классификации шока и определившим принципы его лечения, то второй предложил знаменитое в практической реаниматологии правило «АВС», включающее последовательный комплекс реанимационных действий. Это правило, согласно которому следовало проводить реанимацию, долго доминировало во всем мире, ему обучали и организованные контингенты населения [44, 48].

Современная нормативная база. Большую организационную роль в развитии специальности сыграли приказы МЗ СССР, подготовленные главными специалистами страны А. А. Бунятяном и Р. Н. Лебедевой: № 605 от19.08.1969 г., № 501 от 27.07.1970 г., № 1188 от 29.12.1975 г. и № 841 от 11.06.1986 г. Если в первых из них указывалось о создании реанимационно-анестезиологических отделений, в составе которых развертываются койки для реанимации и интенсивной терапии в больницах от 500 шт. (при наличии не менее 70-100 коек хирургического профиля), то в последующих приказах учитывались реалии структуры и коечной мощности лечебных учреждений страны. В частности, приказ № 841 регламентировал открытие отделений в центральных районных больницах (ЦРБ) при наличии в них не менее 200 коек. Между тем следует подчеркнуть, что ситуация в стране и в системе здравоохранения достаточно быстро меняется и принятые ранее нормативные документы уже не соответствуют новым реалиям жизни. Медицинское сообщество стало более мобильным и информированным, изменяются медицинские технологии диагностики, лечения и формы оказания медицинской помощи. Весьма значимо, что в разных областях огромной страны имеются различия в плотности населения, возрастной структуре, заболеваемости, доступности медицинской помощи и средней продолжительности жизни. Все это привело к мысли, что существующие приказы должны быть адаптированы к условиям отдельного региона или страны [4]. Согласно Федеральному закону от 21.11.2011 г. № 323-ФЗ «Об основах охраны здоровья граждан Российской Федерации» с 1 января 2013 г. медицинская помощь организуется и оказывается в соответствии с порядками оказания медицинской помощи, обязательными для исполнения на территории Российской Федерации всеми медицинскими организациями, а также на основе стандартов медицинской помощи, за исключением медицинской помощи, оказываемой в рамках клинической апробации. В настоящее время в области интенсивной терапии вся повседневная деятельность отделений выстраивается в соответствии с Порядками № 909н и № 919н от 12.11.2012 г. и 15.11.2012 г., которые регламентируют помощь детям и взрослому населению по профилю «анестезиология и реаниматология».

В дополнение к данным порядкам МЗ РФ выпустило Приказ № 625н от 14.09.2018 г., в котором отмечены пункты, вступающие в действие с 13.11.2018 г. Наиболее важные изменения касаются участия анестезиологов-реаниматологов в паллиативной медицинской помощи, создания дистанционных консультативных центров и оказания медицинской помощи не только на основе стандартов, но и с учетом клинических рекомендаций (протоколов лечения).

Роль интенсивной терапии в современной клинической медицине. Современное клиническое значение анестезиологии-реаниматологии и интенсивной терапии и ее роль среди клинических дисциплин трудно переоценить. Вернее, трудно представить современный стационар без ОРИТ. Сегодня это самостоятельная специальность, имеющая в своем составе многотысячную армию, как правило, активных специалистов врачебного и сестринского звеньев с набором жизнеспасающих технологий и служащих опорой коллегам в своем лечебном учреждении, регионе, а в некоторых случаях - за его пределами. Важно подчеркнуть, что многие из врачей, представителей различных специальностей (пульмонологи, кардиологи, хирурги, акушеры-гинекологи, неврологи и др.), используют в своей практике приемы интенсивной терапии. Определим краеугольные камни, характеризующие современную интенсивную терапию.

Численность и квалификация персонала. На сегодня в медицинских организациях МЗ РФ имеется около 58 тысяч ставок врачей анестезиологов-реаниматологов, из которых почти 52 тысячи распределены между 31 тысячей физических лиц. Отметим, что за последние 10 лет численность анестезиологов-реаниматологов в России возросла на 7500 [5]. Сколько из них работает в ОРИТ - таких данных нет. Между тем мы отдаем себе отчет в том, что ситуация в отношении квалификации персонала и 
обеспеченности врачами и медсестрами различных отделений достаточно разнородная. Но если рассматривать сложившуюся динамику или устойчивый тренд, мы можем констатировать заметные перемены, которые наблюдаем за период формирования специальности. Во-первых, как бы мы не сетовали на существующий в отдельных местах дефицит персонала, корпус специалистов, работающих в отделениях реанимации и в службе, сложился. Во-вторых, на основании существующего законодательства можно утверждать, что подавляющее большинство специалистов ОРИТ имеют соответствующие сертификаты. В-третьих, появление новых технологий гемодинамической и респираторной поддержки, аппаратного оснащения, мониторинга, биомаркеров, лекарственных средств неминуемо отразилось на квалификации персонала, его умении, навыках и представлениях о самой специальности. В целом мы полагаем, профессиональный рост персонала от момента ее дебюта налицо, однако существующая система образования, включая постдипломную подготовку, нас, безусловно, сегодня не удовлетворяет, как и квалификация специалиста. Его становление и дальнейшее развитие зависят от удовлетворенности самого клинического ординатора или стажированного врача. Внедренная в жизнь система непрерывного медицинского образования прогрессивна по своей сути, но малореализуема. Ее дальнейшее развитие тормозится огромными территориями страны, ведомственностью медицины, дефицитом специалистов в ЦРБ.

Стратегия ведения пациентов в ОРИТ: от чего ушли вчера и отказываемся сегодня. Давайте вспомним, что было на заре интенсивной терапии: существование широких показаний для проведения инфузионной терапии, обязательное добавление к кристаллоидам витаминов, гормонов, антигистаминных средств, диуретиков, сердечных гликозидов, «знаменитой» кокарбоксилазы. Настоящая интенсивная терапия не мыслилась без гипербарической оксигенации, плазмафереза, гемосорбции, спленоперфузии, непрямого электрохимического окисления крови гипохлоритом натрия, озонотерапии, ультрафиолетового и лазерного облучение крови. Сегодня мы можем утверждать, что излишне либерально трактовались показания для трансфузии компонентов крови - эритроцитарной массы (ЭМ) и свежезамороженной плазмы [8]. Хорошим тоном считалось использование различных иммунокорректоров в послеоперационном периоде и при ряде критических состояний. Реаниматологи того времени были преисполнены чувства великой тайны, известной только им самим, а сами технологии и препараты, число которых постоянно возрастало, способными к возвращению жизни умирающих от травм и болезней. Однако что из перечисленного используется рутинно сейчас? Большинство из применяемых тогда медицинских технологий и лекарственных средств безвозвратно ушли в историю медицины. В результате формируется прагматичная логика «клинической пользы от...», набирает силу понимание стоимостной оценки диагностики и лечения. Достаточно вспомнить истории с активированным протеином С (АПС), растворами крахмалов, иммуноглобулинами, селеном. Более 10 лет назад J.-L. Vincent et al., проведя анализ 72 проспективных контролируемых рандомизированных исследований (ПРКИ), посвященных реанимационным больным, показали, что только в 10 из них был получен позитивный результат [37]. В четырех ПРКИ показано снижение летальности при режимах протективной ИВЛ, по одному - от введения АПС, низких доз гидрокортизона при септическом шоке, высоких доз иммуноглобулина $\mathrm{G}$ у пациентов с сепсисом при Sepsis Score $\geqslant 20$ баллов, гемофильтрации при внебольничной остановке сердца, использования измерения $\mathrm{pH}$ подслизистой желудка в качестве ориентира при инфузионной терапии, инвазивного забора биоматериала при подозрении на вентилятор-ассоциированную пневмонию. Зададим себе вопрос: а что из перечисленного сохранило значимость до настоящего времени и используется сейчас? С некоторыми ограничениями остались только одно лекарственное средство и одна реанимационная технология искусственной респираторной поддержки: низкие дозы гидрокортизона при септическом шоке и протективная ИВЛ в случае развития острого респираторного дистресс-синдрома. Причем в обоих случаях со стороны немалого количества специалистов имеются сомнения в их обязательном применении. К аналогичным пессимистическим выводам пришел и J. Marshall, анализируя результаты более 100 исследований, посвященных оценке эффективности иммуномодуляции и иммунной заместительной терапии при сепсисе [32].

Меньше агрессии в отношении критически больного пациента. Логичным продолжением обозначившейся эволюции по ведению критических больных следует признать снижение агрессивности в диагностике, лечении и профилактике возможных осложнений. В этом отношении неким объединяющим моментом послужила публикация в 2013 г. M. Koх и P. Pickkers в JAMA, призывающая к рациональному ограничению интенсивной терапии при критических состояниях [29]. В качестве научной аргументации такой стратегии служил анализ 11 направлений интенсивной терапии, в котором показаны клинические преимущества минимальной достаточности воздействия, вред или отсутствие позитивного эффекта от попыток быстрого достижения «нормы». Конкретным обоснованием являлись примеры повышения летальности при проведении высокообъемной ИВЛ, достижении высоких значений $\mathrm{PaO}_{2}$, стратегии либеральной инфузии и трансфузии компонентов крови, высокодозной терапии вазопрессорами и глюкокортикостероидами. Оказалось, что вред могут приносить и высокий уровень калорической поддержки, глубокая седация и строгий контроль гликемического профиля посредством инсулина. В хорошо организованных 
ПРКИ не получено доказательств пользы при ежедневном рентгенологическом контроле органов грудной клетки у пациентов на ИВЛ, инвазивного гемодинамического контроля с помощью катетера Свана - Ганса, длительного применения антибиотиков в лечении инфекций, высокообъемной гемофильтрации. И, как оказалось, на этом аргументы авторов не были исчерпаны. На целесообразность следования обозначенной стратегии в ОРИТ указывают все новые и новые исследования, результаты которых были опубликованы позднее. Они касаются рестрикции к трансфузии ЭМ. Так, при использовании в качестве триггера к трансфузии ЭМ снижения содержания гемоглобина менее 70 г/л наблюдалось уменьшение риска смерти, рецидива кровотечения, отека легких и развития нозокомиальных инфекций (НИ) [41]. В унисон с предыдущими исследователями S. Patel et al. отмечают, что с трансфузией каждой дополнительной дозы ЭМ повышается риск смерти, органно-системной дисфункции и острого повреждения легких [38]. Аналогичные заключения в пользу сокращения объема инфузии были сделаны и при септическом шоке [10, 23, 33]. Особую роль в снижении агрессивности в отношении пациента сыграли новые технологии респираторной поддержки, позволяющие в части случаев избежать проведения ИВЛ или сократить ее длительность [11, 13, 22]. О достаточности 4-дневных курсов антибиотикотерапии при адекватно оперированной интраабдоминальной инфекции свидетельствует пионерское исследование 2015 г. [39].

Мультидисциплинарный подход при ведении пациентов. Характер патологии пациентов, госпитализированных в отделение реанимации, может требовать привлечения к процессу ведения в ОРИТ специалистов самого различного профиля. Речь идет не только об основной патологии, когда привлечение к оказанию помощи хирургов, неврологов, терапевтов, травматологов является рутинным и вполне понятным. У «хронических реанимационных больных» существует необходимость участия в их ведении кинезитерапевтов, клинических фармакологов и других специалистов [31, 40]. Появившиеся единичные примеры в наших отделениях реанимации свидетельствуют об однозначной целесообразности применения на практике данной организационной технологии. Между тем на настоящий момент мы не знаем как потребности в подобных специалистах в силу отсутствия эпидемиологических исследований по России, так и структуры этой службы. По крайней мере, имеющиеся нормативные документы не призывают нас к созданию групп ранней реабилитации. Безусловно, необходимость мультидисциплинарного подхода возникает и будет возникать в крупных многопрофильных стационарах, где более реально появление «хронически реанимационного больного» и узкого специалиста, приносящего ему пользу.

Роль балльных измерительных систем (икал). Сегодня никого не удивить использованием шкал на практике и в научных исследованиях. Внедрение в повседневную практику немалого количества реанимационных отделений различных шкал представляется полезным в нескольких отношениях [28, 47]: 1) понимании тяжести поступающих в ОРИТ пациентов, классифицируемых по тяжести общего состояния (АРАCHЕ-II) или органной дисфункции (SOFA); 2) оценке динамики ответа на ту или иную технологию интенсивной терапии; 3) определении трудозатрат персонала; 4) оценке степени седации при возникновении делирия или у пациентов на ИВЛ; 5) определении уровня мобилизуемости пациента.

Диалог с родственниками. Время выработало определенные клише в объяснении причины попадания в ОРИТ. Однако в целом эта крайне важная процедура была пущена на самотек. Наибольшее количество жалоб, поступающих на работников здравоохранения, связано с отсутствием общения с врачом или поверхностным с ними разговором. Мы считаем, что диалог с родственниками пациента, госпитализированного в ОРИТ, объяснение природы критического состояния и осторожное заключение в отношении прогноза имеет весьма важное значение. Разговором с родственниками ни в коем случае нельзя пренебрегать, как бы неприятен он не был. К нему может быть привлечен и профильный специалист. В случае неутешительного исхода роль реаниматолога при собеседовании представляется ключевой [19, 25]. На сегодняшний день важным подспорьем в случае просьбы о посещении могут служить информационно-методическое письмо «О правилах посещения родственниками пациентов в отделениях реанимации и интенсивной терапии (реанимации)» и «Форма памятки для посетителей». Они выпущены 30.05.2016 г. и разработаны внештатными специалистами Министерства здравоохранения РФ и специалистами федеральных государственных медицинских учреждений. В настоящее время на рассмотрении в Госдуме находится законопроект № 359335-7 о внесении изменения в часть 1 статьи 79 Федерального закона «Об основах охраны здоровья граждан в Российской Федерации» (в части посещения пациентов в отделении медицинской организации, оказывающем реанимационные мероприятия).

Интенсивная терапия и ближайшее будущее. Проблемы, которые предстоит решать. Вполне очевидно, что новое время, популяционные перемены в обществе, инновационные технологии, изменения в системе оказания медицинской помощи неизбежно отразятся и на организации помощи при критических состояниях. В данном разделе публикации мы бы хотели избежать излишних фантазий, а обсудить видимые проблемы, которые возникают перед специалистами и потребуют решения в обозримом будущем. Что, на наш взгляд, должно быть преодолено, поскольку не соответствует общей тенденции развития в оказании медицинской помощи? 
Затраты на интенсивную терапию. Появившиеся с начала 50-х годов ХХ в. ОРИТ стали в настоящее время неотъемлемым структурным элементом современного стационара. Благодаря их организации и внедрению современных технологий интенсивной терапии и мониторинга удалось улучшить результаты лечения при тяжелой термической и механической травме, отдельных нозологиях, сопровождающихся развитием шока, синдрома полиорганной недостаточности. Мы выражаем надежду, что уже в обозримом будущем будет более адекватно решаться проблема финансирования службы и работы отделений реанимации. Система финансирования лечебных учреждений на основе клинико-статистических групп (КСГ) с 2015 г. стала внедряться на территории России. Между тем, согласно заложенным в ее основание принципам, она не учитывает отдельно материальные затраты на пребывание пациента в ОРИТ, растворяя их внутри конкретной КСГ. При этом полностью отсутствует информация, каким образом и насколько адекватно рассчитана стоимость реанимационного этапа. Очевидно, что сегодня каждое лечебное учреждение решает эту проблему ситуационно, опираясь на свои материальные возможности, а в некоторых случаях в качестве решающего фактора выступают сложившиеся отношения между главным врачом и ТФОМС, региональным Минздравом или Управлением здравоохранения города и руководителем отделения реанимации.

Эффективная интенсивная терапия стоит недешево. Известно, что уже в конце 80-х годов прошлого столетия денежные затраты на содержание ОРИТ в отдельных странах достигали 1\% от национального ВВП, составляя на уровне лечебных учреждений $20 \%$ от всех общебольничных расходов [26]. Они включают оплату труда персонала, приобретение расходных материалов и лекарственных средств, сервисные услуги на поддержание в работоспособном состоянии дорогостоящего медицинского и прочего оборудования, а также всей инфраструктуры отделения в целом. В настоящее время эти расходы заметно возросли, средняя стоимость койко-дня в ОРИТ европейских стран колеблется от 900 евро в Испании до 3300 евро в Дании [15].

Итак, нет никакого сомнения в том, что система финансирования ОРИТ в России нуждается в совершенствовании. Возможность разрешения проблемы оптимального финансирования реанимационного пациента во многом зависит от правильности показаний для пребывания в ОРИТ, а в ряде случаев и в оценке ответа на проводимую интенсивную терапию. Проводимые сегодня аудиты региональных министерств здравоохранения, Росздравнадзора, ТФОМС и других контролирующих организаций не касаются данного вопроса. Мы полагаем, что в будущем проблема разрешится при изменении модели оказания помощи в стационаре: создании 3-уровневой модели оказания неотложной помощи или промежуточных отделений, где больше ухода, чем реанимации и интенсивной терапии $[17,50]$.
Кадровый потенциал: нужен новый тип специалистов анестезиологов-реаниматологов. Начнем с проблемы подготовки кадров, которые, как известно, определяют все. Следует признать, что сегодня двухгодичная подготовка в клинической ординатуре, закладывая базовые навыки, не дает необходимых знаний для полноценной самостоятельной работы в клинической больнице или НИИ. В этом отношении мы полностью солидарны с позицией, обозначенной в недавней публикации академика РАН Ю. С. Полушина [5]. Количество компетенций анестезиолога-реаниматолога, необходимых для многопрофильной клиники и небольшой ЦРБ, весьма значимо отличается. Причем врач ЦРБ чем дальше, тем более замыкается на весьма ограниченном количестве клинических ситуаций, нозологий и операций, теряя свой еще неполный профессиональный багаж, сохраняя некую его крупную частицу. Рост в поливалентных отделениях реанимации числа соматических больных с учетом старения населения требует увеличения от организации количества ОРИТ или создания таковых в больницах, не имеющих хирургических отделений. Между тем структура пациентов данных отделений диктует необходимость совсем другого уровня знаний в области кардиологии, неврологии и пульмонологии, другого врача. Жизненный опыт показывает, что и в PAO с достаточно мощной анестезиологией руководители подразделений определяют на работу в палату реанимации весьма ограниченный круг врачей, которые пригодны по уровню своих знаний, психологической устойчивости и физическим возможностям. В свете вышеизложенного нам представляется два пути в разрешении проблемы. Первый из них состоит в увеличении сроков подготовки врачей для крупных клиник до 3 лет с возможностью дежурств после 2 лет обучения в лечебно-профилактических учреждениях (ЛПУ) более низкого уровня. Второй, менее благоприятный, но более реальный, - нелегальное «маневрирование» на местах программой двухлетнего обучения с отказом от некоторых предусмотренных ФГОС блоков. Однако следует иметь в виду, что ступая на эту стезю, мы неизбежно навлекаем на себя гнев вузовской администрации и попадаем в конкурентные взаимоотношения с преподаванием других дисциплин, в частности организации здравоохранения и педагогики. Хотя практическое значение преподносимых ими знаний для ординатора анестезиолога-реаниматолога с неадекватным по времени периодом подготовки вызывает большие сомнения.

Ультразвуковая диагностика. Польза в ОРИТ от ургентной ультразвуковой диагностики ни у кого сомнений не вызывает, как и соответствующие компетенции реаниматолога. Новое поколение портативных аппаратов позволяет применять их в экспресс-режиме для катетеризации центральных вен, регионарных блокад, диагностики природы шока, ответа на инфузию, характера острого легочного повреждения [18, 27]. Такая диагностика создает 
условия для быстрого разрешения критического состояния и выбора терапии. Очень отрадно, что энтузиасты данной технологии среди реаниматологов появились и в России. Однако главным сдерживающим моментом в РФ служит необходимость выполнения программы 2-годичной ординатуры, поскольку без сертификата специалиста выполненная врачом-реаниматологом ультразвуковая диагностика не может быть принята во внимание. Определенное негативное значение имеет и дефицит времени в период обучения в ординатуре. Таким образом, круглосуточное проведение ультразвуковой диагностики в ОРИТ возможно только в крупных ЛПУ, использование технологии в ЦРБ анестезиологу-реаниматологу доступно опять только на нелегальной основе. Хочется верить, что данная весьма полезная для пациентов ОРИТ неинвазивная технология в обозримом будущем легально расширит границы своего применения.

Персонализированная терапия. Мы уже отмечали, что результаты множества проспективных рандомизированных исследований, касающиеся лекарственных новшеств, принесли в основном разочарования. Возникший скепсис связан с тремя основными причинами: гетерогенностью пациентов ОРИТ, генетической детерминацией ответа на повреждение, разноликостью больных в различные фазы болезни. По этой причине проводимое лечебное воздействие должно опираться не на жесткие клинические рекомендации, а на принципы ведения больного, основанные на физиологии и оценке ответа на тот или иной метод интенсивной терапии [18]. Например, стартовая интенсивная инфузионная терапия сепсиса должна быть индивидуальной, с учетом локализации очага, возраста, сопутствующей патологии, динамики температурного градиента, диуреза и, главное, реакции на нее клинических или инструментальных критериев (частота сердечных сокращений, систолическое артериальное давление, диаметр нижней полой вены, пульсовое давление). Помимо инфузионной терапии, индивидуально, с учетом нарушений гомеостаза и под конкретную ситуацию должны подбираться и другие ее компоненты (сбалансированные растворы, альбумин, ЭМ, свежезамороженная плазма), как и прочие составляющие интенсивной терапии (гепарины, препараты для седации, гидрокортизон). Если выбор гепарина и его режима дозирования начинает принципиально меняться при снижении клиренса креатинина менее 30 мл/мин, то оптимальный препарат для седации/адаптации к респиратору будет определяться конкретной клинико-лабораторно-инструментальной ситуацией. Гораздо сложнее обстоит ситуация с назначением гидрокортизона при септическом шоке, поскольку чувствительность ГКС-рецепторов может меняться в ходе течения патологического процесса, отсутствует и единое понимание рефрактерности шока $[35,49]$. Эта же логика персонализации распространяется как на методы респираторной поддержки, так и на устанавливаемые ежедневно параметры проведения ИВЛ.
Ранняя мобилизация. Ранняя мобилизация на этапе ОРИТ должна стать частью ежедневной рутинной практики. Вместе с тем эта технология требует изменения психологии всех участвующих в процессе ранней реабилитации, внедрения в практику нового инструментария, а главное, создания команды, предметно занимающейся ранней реабилитацией. Ее жизнеспособность, помимо специально подготовленных кадров, связана с отладкой системы финансирования через ТФОМС и КСГ. В целом подавляющее большинство реаниматологов согласны с проведением ранней мобилизации на этапе ОРИТ, и ее необходимость в основном сомнений не вызывает. Можно привести немало доводов в ее пользу: снижение риска осложнений, связанных с постельным режимом, мышечной слабости, возникшей в ОРИТ в связи с заболеванием или последствиями эффекта некоторых препаратов (глюкокортикостероиды, бензодиазепины, миорелаксанты), улучшение функционального статуса, снижение частоты делирия, уменьшение длительности пребывания в ОРИТ, в стационаре, а также годичной летальности. Наряду с организационными основами внедрения в практику, значимыми остаются вопросы типа кратности и длительности проведения мобилизации, присутствия сопутствующей седации, выявления популяции пациентов с функциональным ответом на раннюю мобилизацию [20, 40].

Профилактика инфекиионных осложнений. Профилактика инфекционных осложнений в период пребывания в ОРИТ стала иметь одно из главных значений в связи с несколькими обстоятельствами: сокращением летальности в остром периоде критических состояний, увеличением числа пациентов на ИВЛ, ростом резистентности к антибиотикам возбудителей НИ $[6,7]$. ОРИТ оказались неким «горячим цехом резистентности», где используются спасительные, но агрессивные технологии мониторинга и лечения, которые могут сопровождаться развитием тяжелых осложнений, включая инфекционные. Объективными причинами для роста количества НИ, помимо снижения летальности, служат увеличение продолжительности жизни при тяжелых хронических заболеваниях, расширение показаний к хирургическим вмешательствам у лиц пожилого и старческого возраста, имеющих, как правило, различную по степени выраженности фоновую патологию. Сам же пациент оказался новой искусственно созданной нишей для микроорганизмов в относительно изолированном пространстве. Для таких пациентов характерна иммунная дисфункция, выражающаяся в повреждении механизмов антиинфекционной защиты. По данным различных источников, средняя частота развития НИ в ОРИТ варьирует от 25 до $49 \%$, что в 5 раз и более превышает таковую в других отделениях стационара $[1,9]$.

В качестве субъективной причины высокого уровня потребления антибактериальных препаратов в ОРИТ следует отметить неумение ряда специалистов отличить колонизацию от инфекции, 
боязнь пропустить жизнеугрожаемый процесс инфекционной природы, непоколебимую веру в продолжительную антибиотикопрофилактику, нежелание проведения режима деэскалации, излишне длительные курсы терапии. Наибольший эффект в предупреждении инфекций принесли контроль над потреблением антибиотиков и совершенствование методов обработки рук персоналом [12, 24, 36, 42]. В итоге реаниматологи будут вынуждены заниматься подходами к оптимальной обработке не только своих рук, но и самого пациента, более обоснованно относиться к необходимости каждого дня пребывания в ОРИТ и антимикробной терапии. И в этом отношении будет полезным структурирование отделений реанимации на уровни или создание отделения промежуточной медицинской помощи.

Венская декларащия по безопасности в отделениях реанимации Европейского общества интенсивной медииины (ESICM). Спустя достаточно длительный период времени существования ОРИТ возникло понимание необходимости обеспечения безопасности в момент пребывания в данном подразделении критического больного. Стало очевидно, что пациент в критическом состоянии более уязвим в отношении различного рода осложнений в силу уже наличия у него органно-системных расстройств и вынужденной необходимости использования агрессивных методов лечения, таких как катетеризация артериальных и венозных стволов, ИВЛ, нейроаксиальные блокады, заместительная почечная терапия, сорбция, инфузия компонентов крови, внутривенное введение потенциально опасных фармакологических средств. Таким образом, обоснованно полагают, что вся лечебно-диагностическая работа в ОРИТ должна проводиться и с созданием условий безопасности для пациента. В итоге появилась однозначная необходимость в повседневной практике следовать Венской декларации по безопасности Европейского общества интенсивной медицины (ESICM) от
2009 г. [34]. Авторы документа призывают признать существование «эпидемии ошибок» и то, что без повышения безопасности в ОРИТ невозможно улучшить качество оказания помощи в стационаре в целом. В свою очередь построение среды безопасности в ОРИТ основано на следующих положениях.

- Формирование особого менталитета персонала.

- Постоянное обучение всего коллектива ОРИТ.

- Работа по клиническим рекомендациям (протоколам).

- Внутренний аудит (разбор) осложнений.

- Адекватный микроклимат в коллективе.

- Оптимизация нагрузки на персонал.

\section{Заключение}

Роль медицины критических состояний возрастает. Можно прогнозировать рост числа коек интенсивного этапа оказания помощи в стационарах. Рано или поздно мы будем вынуждены структурировать ее оказание в ОРИТ различного уровня в зависимости от тяжести состояния пациентов и целесообразности проведения лечения в полном объеме. Существующая длительность подготовки анестезиолога-реаниматолога во многом не соответствует потребностям современной медицины. Возникла необходимость сертификации различного уровня в зависимости от будущего места работы специалиста. Между тем следует констатировать более рациональное отношение врачей к использованию многих лечебно-диагностических методов и лекарственных средств. Среди обозримых перспектив развития специальности следует выделить определение ее финансирования в соответствии с затратами, создание в медицинских организациях группы ранней мобилизации и реабилитации, улучшение профилактики инфекционных осложнений.

Конфликт интересов. Автор заявляет об отсутствии у него конфликта интересов.

Conflict of Interests. The author state that he has no conflict of interests.

\section{ЛИТЕРАТУРА}

1. Гайфутдинов Е. А. Роль управления антимикробной терапией в службе реанимации и интенсивной терапии многопрофильного стационара // Клиническая микробиология и антимикробная химиотерапия. - 2018. T. 20, № 2. - C. 132-140.

2. Золотокрылина Е. С. Постреанимационная болезнь // Анестезиология и реаниматология. - 2000. - Т. 6. - С. 68-73.

3. Интенсивная терапия: национальное руководство: в 2 т. / под ред. Б. Р. Гельфанда, А. И. Салтанова. - М.: ГЭОТАР-Медиа, 2009. - Т. І. - 960 с

4. Недашковский Э. В. В кн.: Интенсивная терапия: национальное руководство: в 2 т. / под ред. Б. Р. Гельфанда, А. И. Салтанова. - М.: ГЭОТАР-Медиа. - 2009. - Т. І. - С. 29-49.

5. Полушин Ю. С. Проблемные вопросы анестезиолого-реанимационной помощи // Вестник анестезиологии и реаниматологии. - 2019. - Т. 16, № $1 .-$ C. 5-12.

\section{REFERENCES}

1. Gayfutdinov E.A. Role of antimicrobial therapy management in the intensive care of multi-specialty hospital. Klinicheskaya Mikrobiologiya i Antimikrobnaya Khimioterapiya, 2018, vol. 20, no. 2, pp. 132-140. (In Russ.)

2. Zolotokrylina E.S. Post-resuscitation disease. Anesteziologiya i Reanimatologiya, 2000, vol. 6, pp. 68-73. (In Russ.)

3. Intensivnaya terapiya. Natsionalnoye rukovodstvo. [Intensive care. National guidelines]. 2 volumes, B.R. Gelfand, A.I. Saltanov, eds. Moscow, GEOTAR-Media Publ., 2009, vol. 1, 960 p.

4. Nedashkovskiy E.V. In: Intensivnaya terapiya. Natsionalnoye rukovodstvo. [Intensive care. National guidelines]. 2 volumes, B.R. Gelfand, A.I. Saltanov, eds. Moscow, GEOTAR-Media Publ., 2009, vol. 11, no. 1, pp. 29-49.

5. Polushin Yu.S. Topical issues of anesthesiology and intensive care. Vestnik Anesteziologii I Reanimatologii, 2019, vol. 16, no. 1, pp. 5-12. (In Russ.) 
6. Руднов В. А., Бельский Д. В., Дехнич А. В., исследовательская группа РИОРИТа. Инфекции в ОРИТ России: результаты национального многоцентрового исследования // Клин. микробиол. антимикроб. химиотер. 2011. - T. 13, № 4. - C. 294-303.

7. Сухорукова М. В., Эйдельштейн М. В., Склеенова Е. Ю. и др. Антибиотикорезистентность нозокомиальных штаммов Enterobacteriaceae в стационарах России: результаты многоцентрового эпидемиологического исследования МАРАФОН в 2011 - 2012 // Клин. микробиол. антимикроб. химиотер. - 2014. - Т. 16, № 4. - С. 254-265.

8. Шанин Ю. Н., Волков Ю. Н., Костюченко А. Л. и др. Послеоперационная интенсивная терапия. - Л.: Медицина, 1978. - 224 с.

9. Яковлев С. В., Суворова М. П., Белобородов В. Б. и др. Распространенность и клиническое значение нозокомиальных инфекций в лечебных учреждениях России: исследование ЭРГИНИ // Антибиотики и химиотерапия. - 2016. - Т. 61, № 5-6. - С. 32-42.

10. Angus D., Barnato A., Bell D. et al. A systematic and meta-analysis of early goal-directed therapy for septic shock: the ARISE, ProCESS and ProMiSe investigators // Intens. Care Med. - 2015. - Vol. 41. - P. 1549-1560.

11. Antonelli M., Conti G., Rocco M. et al. A Comparison of noninvasive positive-pressure ventilation and conventional mechanical ventilation in patients with acute respiratory failure // N. Engl. J. Med. - 1998. - Vol. 339. - P. 429-435.

12. Barlam T., Cosgrove S., Abbo L. et al. Implementing an antibiotic stewardship program: Guidelines by the Infectious Disease Society of America and Society for Healthcare Epidemiology of America // Clin. Infect. Dis. - 2016. - Vol. 62 № 10. - P. e51-e77.

13. Barr J., Fraser G. L., Puntillo K. et al. Clinical practice guidelines for the management of pain, agitation, and delirium in adult patients in the intensive care unit // Crit. Care Med. - 2013. - Vol. 41. - P. 263-306.

14. Berthelsen P., Cronovist M. The first intensive care unit in the world: Copenhagen 1953 // Acta Anaesth. Scand. - 2003. - Vol. 47. - P. 1190-1195.

15. Bittner M.-J., Donelly M., van Zanten A. et al. How is intensive care reimbursed? Review of eight European countries // Ann. Intens. Care. - 2013. - Vol. 3. - P. 37

16. Calvin J., Habet K, Parrillo J. Critical care in the United States. Who are we and how did we get here? // Crit. Care Clin. - 1997. - Vol. 13, № 2. - P. 363-376.

17. Capuzzo M., Volta C., Tassinati T. et al. Hospital mortality of adults admitted to Intensive Care Units in hospitals with and without Intermediate Care Units: a multicenter European cohort study // Crit. Care. - 2014. - Vol. 18. - P. 551.

18. Choong See K., Ong V., Lin Tan Yi et al. Chest radiography versus lung ultrasound for identification of acute respiratory distress syndrome: a retrospective observational study // Crit. Care. - 2018. - Vol. 22. - P. 203.

19. Curtis J., Patrick D., Shannon S. et al. The family conference as a focus to improve communication about end-of-life care in the ICU: opportunities for improvement // Crit. Care Med. - 2001. - Vol. 29. - P. 26-33.

20. Denehy L., Lanphere J., Needham D. Ten reasons why ICU patients should be mobilized early // Intens. Care Med. - 2017. - Vol. 43. - P. 86-90.

21. Feissel M., Michard F., Faller J. P. et al. The respiratory variation in inferior vena cava diameter as a guide to fluid therapy // Intens. Care Med. - 2004. Vol. 30. - P. 1834-1837.

22. Frat J-P., Thille A., Mrrcat A. et al. High-flow oxygen through nasal cannula in acute hypoxemic respiratory failure // N. Engl. J. Med. - 2015. - Vol. 372 № 23. - P. 2185-2196.

23. Hjortrup P., Haase N., Budhaard H. et al. Restricting volumes of resuscitation fluid in adults with septic shock after initial management: the CLASSIC randomised, parallel group, multicentre feasibility trial // Intens. Care Med. 2016. - Vol. 42, № 11. - P. 1695-1705.

24. Honda H., Ohmagari N., Tokuda Y. et al. Antimicrobial stewardship in inpatien settings in the Asia Pacific region: a systematic review and meta-analysis // Clin. Infect. Dis. - 2017. - Vol. 64. - P. 119-126.

25. Hunter J., Goddard C., Rothwell M. et al. A survey of intensive care units visiting policies in the United Kingdom // Anesthesia. - 2010. - Vol. 65. - P. 1101-1105.

26. Jacobs P., Noseworthy T. National estimate of intensive care utilization and cost Canada and the United States // Crit. Care Med. - 1990. - Vol. 18. - P. 1282-1286.

27. Kelmenson D., Quan D., Moss M. What is the diagnostic accuracy of single nerve conduction studies and muscle ultrasound to identify critical illness polyneuromyopathy: a prospective cohort study // Crit. Care. - 2018. - Vol. 22. - P. 342

28. Knaus W., Draper E., Wagner D. et al. APACHE-II: a severity of disease classification system // Crit. Care Med. - 1985. - Vol. 13. - P. 818-829.

29. Kox M., Pickkers P. "Less is more" in critically ill patients. Not too intensive // JAMA Intern. Med. - 2013. - Vol. 10. - P. 1369-1372.
6. Rudnov V.A., Belskiy D.V., Dekhnich A.V., RIORIT research group. Infections in the intensive care units in Russia: results of the national multi-center trial. Clin. Microbiol. Antimicrob. Khimioter., 2011, vol. 13, no. 4, pp. 294-303. (In Russ.)

7. Sukhorukova M.V., Eydelshteyn M.V., Skleenova E.Yu. et al. Antibiotic resistance in nosocomial strains of Enterobacteriaceae in hospitals of Russia: results of multi-center epidemiological study of MARATHON in 2011-2012. Klin. Microbiol. Antimicrob. Khimioter., 2014, vol. 16, no. 4, pp. 254-265. (In Russ.)

8. Shanin Yu.N., Volkov Yu.N., Kostyuchenko A.L. et al. Posleoperatsionnaya intensivnaya terapiya. [Post-operative intensive care]. Leningrad, Meditsina Publ., 1978, 224 p.

9. Yakovlev S.V., Suvorova M.P., Beloborodov V.B. et al. Prevalence and clinical significance of nosocomial infections in the medical units of Russia: ERGIN trial. Antibiotiki i Khimioterapiya, 2016, vol. 61, no. 5-6, pp. 32-42. (In Russ.)

10. Angus D., Barnato A., Bell D. et al. A systematic and meta-analysis of early goal-directed therapy for septic shock: the ARISE, ProCESS and ProMiSe investigators. Intens. Care Med., 2015, vol. 41, pp. 1549-1560.

11. Antonelli M., Conti G., Rocco M. et al. A Comparison of noninvasive positive-pressure ventilation and conventional mechanical ventilation in patients with acute respiratory failure. N. Engl. J. Med., 1998, vol. 339, pp. 429-435.

12. Barlam T., Cosgrove S., Abbo L. et al. Implementing an antibiotic stewardship program: Guidelines by the Infectious Disease Society of America and Society for Healthcare Epidemiology of America. Clin. Infect. Dis., 2016, vol. 62, no. 10, pp. e51-e77.

13. Barr J., Fraser G.L., Puntillo K. et al. Clinical practice guidelines for the management of pain, agitation, and delirium in adult patients in the intensive care unit. Crit. Care Med., 2013, vol. 41, pp. 263-306.

14. Berthelsen P., Cronovist M. The first intensive care unit in the world: Copenhagen 1953. Acta Anaesth. Scand., 2003, vol. 47, pp. 1190-1195.

15. Bittner M.J., Donelly M., van Zanten A. et al. How is intensive care reimbursed? Review of eight European countries. Ann. Intens. Care, 2013, vol. 3, pp. 37.

16. Calvin J., Habet K., Parrillo J. Critical care in the United States. Who are we and how did we get here? Crit. Care Clin., 1997, vol. 13, no. 2, pp. 363-376.

17. Capuzzo M., Volta C., Tassinati T. et al. Hospital mortality of adults admitted to Intensive Care Units in hospitals with and without Intermediate Care Units: a multicenter European cohort study. Crit. Care, 2014, vol. 18, pp. 551.

18. Choong See K., Ong V., Lin Tan Yi et al. Chest radiography versus lung ultrasound for identification of acute respiratory distress syndrome: a retrospective observational study. Crit. Care, 2018, vol. 22, pp. 203.

19. Curtis J., Patrick D., Shannon S. et al. The family conference as a focus to improve communication about end-of-life care in the ICU: opportunities for improvement. Crit. Care Med., 2001, vol. 29, pp. 26-33.

20. Denehy L., Lanphere J., Needham D. Ten reasons why ICU patients should be mobilized early. Intens. Care Med., 2017, vol. 43, pp. 86-90.

21. Feissel M., Michard F., Faller J.P. et al. The respiratory variation in inferior vena cava diameter as a guide to fluid therapy. Intens. Care Med., 2004, vol. 30, pp. 1834-1837.

22. Frat J.P., Thille A., Mrrcat A. et al. High-flow oxygen through nasal cannula in acute hypoxemic respiratory failure. N. Engl. J. Med., 2015, vol. 372, no. 23, pp. 2185-2196.

23. Hjortrup P., Haase N., Budhaard H. et al. Restricting volumes of resuscitation fluid in adults with septic shock after initial management: the CLASSIC randomised, parallel group, multicentre feasibility trial. Intens. Care Med., 2016, vol. 42 , no. 11 , pp. $1695-1705$

24. Honda H., Ohmagari N., Tokuda Y. et al. Antimicrobial stewardship in inpatient settings in the Asia Pacific region: a systematic review and meta-analysis. Clin. Infect. Dis., 2017, vol. 64, pp. 119-126.

25. Hunter J., Goddard C., Rothwell M. et al. A survey of intensive care units visiting policies in the United Kingdom. Anesthesia, 2010, vol. 65, pp. 1101-1105.

26. Jacobs P., Noseworthy T. National estimate of intensive care utilization and cost: Canada and the United States. Crit. Care Med., 1990, vol. 18, pp. 1282-1286.

27. Kelmenson D., Quan D., Moss M. What is the diagnostic accuracy of single nerve conduction studies and muscle ultrasound to identify critical illness polyneuromyopathy: a prospective cohort study. Crit. Care, 2018, vol. 22, pp. 342.

28. Knaus W., Draper E., Wagner D. et al. APACHE-II: a severity of disease classification system. Crit. Care Med., 1985, vol. 13, pp. 818-829.

29. Kox M., Pickkers P. "Less is more" in critically ill patients. Not too intensive. JAMA Intern. Med., 2013, vol. 10, pp. 1369-1372. 
30. Lassen H. C. A. A preliminary report on the 1952 epidemic of poliomyelitis in Copenhagen // Lancet. - 1953. - Vol. 261. - P. 37-41.

31. MacLaren R., Bond C., Martin S. et al. Clinical and economic outcomes of involving pharmacists in the direct care of critically ill patients with infection // Crit. Care Med. - 2008. - Vol. 36. - P. 3184-3189.

32. Marshall J. Why have clinical trials in sepsis failed // Trends in molecular medicine. - 2014. - Vol. 20, № 4. - P. 196-200.

33. Mouncey P., Osborn T., Power G. et al. Trials of early, goal-directed resuscitation for septic shock // N. Engl. J. Med. - 2015. - Vol. 372. - P. 1301-1311.

34. Moreno R., Rhodes A., Donchin Y. Patient's safety in intensive care medicine: the Declaration of Vienna // Intens. Care Med. - 2009. - Vol. 35. - P. 1667-1672.

35. Nandhabalan N., Joannou N., Meadows C. et al. Refractory septic shock: our pragmatic approach // Crit. Care. - 2018. - Vol. 22. - P. 215.

36. O'Neil J. Antimicrobial Resistance: tackling a crisis for the health and wealth of nations // Rev. Antimicrobial. Res. - 2014. - P. 1-16. https://amr-review.org

37. Ospina-Tacson G., Bouchele G., Vincent J.-L. Multicenter, randomized, clinical trials: doomed to fail // Critical Care Med. - 2008. - Vol. 36, №4 - - P. 1113-1122.

38. Patel S., Kidane B., Klingel M. et al. Risks associated with red blood cell transfusion in the trauma population, a meta-analysis // Int. J. Care Injured. 2014. - Vol. 45. - P. 1522-1533.

39. Sawyer R., Claridge J., Nathens A. et al. Trial of short-course antimicrobial therapy for intraabdominal infection // N. Engl. J. Med. - 2015. - Vol. 372. - P. 1996-2005.

40. Schweikert W., Pohlman M., Pohlman A. et al. Early physical and occupational therapy in mechanically ventilated critically ill patients: a randomized controlled trials // Lancet. - 2009. - Vol. 373. - P. 1874-1882.

41. Shalpeter S., Bukley J., Chatterjee S. Impact of more restrictive blood transfusion strategies on clinical outcomes: a meta-analysis and systematic review // Am. J. Med. - 2014. - Vol. 127. - P. 124-131.

42. Spielberg D., Guidos R., Gilbert D. et al. The epidemic of antibiotic-resistant infection: a call to action for the medical community from Infection Diseases Society of America // Clin. Infect. Dis. - 2008. - Vol. 46. - P. 155-164.

43. Theerawit P., Morasert T., Sutherasan Y. Inferior vena cava diameter variation compared with pulse pressure variation as predictors of fluid responsiveness in patients with sepsis // J. Crit. Care. - 2016. - Vol. 36. - P. 246-251.

44. Vincent J-L. Crit Care - where have we been and where are we going? // Crit Care. - 2013. - Vol. 17. - P. S1-S2.

45. Vincent J.-L. Personalized medicine in Intensive Care // ICU Managemen \& Practice. - 2017. - Vol. 17, Is. 1. - https://healthmanagement.org/c/ icu/issuearticle/personalised-medicine-in-intensive-care

46. Vincent J.-L., Thijs L. Critical care in Europe // Crit. Care Clin. - 1997. - Vol. 13, № 2. - P. 245-254.

47. Vincent J.-L., Moreno R., Takala J. et al. The SOFA (Sepsis-related Organ Failure Assessment) score to describe organ dysfunction/failure // Intens. Care Med. 1996. - Vol. 22. - P. 707-710.

48. Weil M., Shubin H. Diagnosis and Treatment of shock. The Williams and Wilkins Company. Baltimore. - 1967. - 327 p.

49. Wong H., Cvijanovich N., Anas N. Developing a critically feasible personalized medicine approach to pediatric septic shock // Am. J. RCCM. - 2015. - Vol. 191 № 3. - P. 309.

50. Zimmerman J. E., Wagner D. P., Knaus W. A. et al. The use of risk predictions to identify candidates for intermediate care units. Implications for intensive care utilization and cost // Chest. - 1995 - Vol. 108 - P. 490-499.

\section{ДЛЯ КОРРЕСПОНДЕНЦИИ:}

\section{Руднов Владимир Александрович}

Уральский государственный медицинский университет,

доктор медищинских наук, профессор.

620028 , г. Екатеринбург, ул. Репина, д. 3.

$M A У$ «КБ № 40»,

заместитель главного врача по анестезиологии

и реанимачии.

620102, г. Екатеринбург, ул. Волгоградская, д. 189.

E-mail:vrudnov@mail.ru
30. Lassen H.C.A. A preliminary report on the 1952 epidemic of poliomyelitis in Copenhagen. Lancet, 1953, vol. 261, pp. 37-41.

31. MacLaren R., Bond C., Martin S. et al. Clinical and economic outcomes of involving pharmacists in the direct care of critically ill patients with infection. Crit. Care Med., 2008, vol. 36, pp. 3184-3189.

32. Marshall J. Why have clinical trials in sepsis failed. Trends in Molecular Medicine, 2014, vol. 20, no. 4, pp. 196-200.

33. Mouncey P., Osborn T., Power G. et al. Trials of early, goal-directed resuscitation for septic shock. N. Engl. J. Med., 2015, vol. 372, pp. 1301-1311.

34. Moreno R., Rhodes A., Donchin Y. Patient's safety in intensive care medicine: the Declaration of Vienna. Intens. Care Med., 2009, vol. 35, pp. 1667-1672.

35. Nandhabalan N., Joannou N., Meadows C. et al. Refractory septic shock: our pragmatic approach. Crit. Care, 2018, vol. 22, pp. 215.

36. O’Neil J. Antimicrobial Resistance: tackling a crisis for the health and wealth of nations. Rev. Antimicrobial. Res., 2014, pp. 1-16. https://amr-review.org

37. Ospina-Tacson G., Bouchele G., Vincent J.-L. Multicenter, randomized, clinical trials: doomed to fail. Critical Care Med., 2008, vol. 36, no. 4, pp. 1113-1122.

38. Patel S., Kidane B., Klingel M. et al. Risks associated with red blood cell transfusion in the trauma population, a meta-analysis. Int. J. Care Injured, 2014, vol. 45, pp. 1522-1533.

39. Sawyer R., Claridge J., Nathens A. et al. Trial of short-course antimicrobial therapy for intraabdominal infection. N. Engl. J. Med., 2015, vol. 372, pp. 1996-2005.

40. Schweikert W., Pohlman M., Pohlman A. et al. Early physical and occupational therapy in mechanically ventilated critically ill patients: a randomized controlled trials. Lancet, 2009, vol. 373, pp. 1874-1882.

41. Shalpeter S., Bukley J., Chatterjee S. Impact of more restrictive blood transfusion strategies on clinical outcomes: a meta-analysis and systematic review. Am. J. Med., 2014, vol. 127, pp. 124-131

42. Spielberg D., Guidos R., Gilbert D. et al. The epidemic of antibiotic-resistan infection: a call to action for the medical community from Infection Diseases Society of America. Clin. Infect. Dis., 2008, vol. 46, pp. 155-164.

43. Theerawit P., Morasert T., Sutherasan Y. Inferior vena cava diameter variation compared with pulse pressure variation as predictors of fluid responsiveness in patients with sepsis. J. Crit. Care, 2016, vol. 36, pp. 246-251.

44. Vincent J.L. Crit Care - where have we been and where are we going? Crit. Care, 2013, vol. 17, pp. S1-S2.

45. Vincent J.L. Personalized medicine in Intensive Care. ICU Management \& Practice, 2017, vol. 17, is. 1. https://healthmanagement.org/c/icu/issuearticle/pe rsonalised-medicine-in-intensive-care

46. Vincent J.L., Thijs L. Critical care in Europe. Crit. Care Clin., 1997, vol. 13, no. 2, pp. 245-254

47. Vincent J.L., Moreno R., Takala J. et al. The SOFA (Sepsis-related Organ Failure Assessment) score to describe organ dysfunction/failure. Intens. Care Med., 1996, vol. 22, pp. 707-710.

48. Weil M., Shubin H. Diagnosis and Treatment of shock. The Williams and Wilkins Company. Baltimore. 1967, 327 p.

49. Wong H., Cvijanovich N., Anas N. Developing a critically feasible personalized medicine approach to pediatric septic shock. Am. J. RCCM, 2015, vol. 191, no. 3 , pp. 309

50. Zimmerman J.E., Wagner D.P., Knaus W.A. et al. The use of risk predictions to identify candidates for intermediate care units. Implications for intensive care utilization and cost. Chest, 1995, vol. 108, pp. 490-499.

\section{FOR CORRESPONDENCE:}

\section{Vladimir A. Rudnov}

Ural State Medical University,

Doctor of Medical Sciences, Professor.

3, Repina St., Yekaterinburg, 620028.

Municipal Clinical Hospital no. 40,

Deputy Head Doctor for Anesthesiology and Intensive Care.

189, Volgogradskaya St.,

Yekaterinburg, 620102.

Email:vrudnov@mail.ru 